\title{
TRADUCCIÓN
}

\section{"Un cerdo inconformista": el ensayo de Wang Xiaobo sobre la granja orwelliana de la Revolución Cultural ${ }^{1}$}

\author{
"A maverick pig": Wang \\ Xiaobo's essay on the Orwellian \\ farm of the Cultural Revolution
}

\author{
ISMAEL A. MAÍLLO \\ Universidad de Salamanca, España
}

Resumen: El ensayo "Un cerdo inconformista", del escritor chino de culto Wang Xiaobo (1952-1997), fue publicado por primera vez en 1996 en la revista 三联生活周刊 [Life Weekly]. En él se describen las aventuras de un cerdo, mascota de unos jóvenes instruidos, que es

Recepción: 31 de octubre de 2019. / Aceptación: 29 de enero de 2020.

${ }^{1}$ Esta traducción es una versión corregida de la que incluí en mi tesis de doctorado publicada en 2019: Wang Xiaobo, un ensayista revolucionario (traducción y estudio de sus ensayos más representativos). Salamanca: Ediciones Universidad de Salamanca. https://doi.org/10.14201/0VI0441 
distinto a los demás: un cerdo ágil, vivaracho e inteligente que se las arregla para salirse con la suya. El cerdo es considerado una lacra por los lugareños.

Palabras clave: Wang Xiaobo; Revolución Cultural; ensayo; literatura china; literatura contemporánea.

Abstract: "A Maverick Pig" is an essay by Chinese cult writer Wang Xiaobo (1952-1997) that was first published in 1996 in 三联生活周 刊 [Life Weekly] magazine. It describes the adventures of a pig-the pet of educated young men-which is different from the others: an agile, vivacious, and intelligent creature that manages to get away with things. For the local people, the pig is a scourge.

Keywords: Wang Xiaobo; Cultural Revolution; essay; Chinese literature; contemporary literature.

\section{Introducción}

Wang Xiaobo (1952-1997) irrumpió en la escena literaria china en la década de 1990 y, cual ventolera, falleció pocos años más tarde y dejó a su creciente grupo de seguidores en estado de conmoción. En ese corto espacio de tiempo fue increíblemente prolífico, ya que trabajaba casi sin descanso en su estudio de Shunyi, en el extrarradio de Beijing. Tras su inesperada muerte a los 45 años de edad, sus obras alcanzaron una enorme popularidad y se transmutaron en un fenómeno cultural entre las clases medias y en particular en los círculos universitarios.

Tanto sus obras de ficción como su producción ensayística giran en torno a la Revolución Cultural (1966-1976), que marcó su vida de manera indeleble. Ahora bien, lo que hace diferente a Wang Xiaobo de otros autores es su tratamiento irreverente y desprejuiciado de ese periodo traumático. Con humor negro y una retórica extravagante, teniendo presente el punto de vista del individuo y el papel de los intelectuales, se desmarca de las dos interpretaciones de la Revolución Cultural que predo- 
minaban en la literatura china tras la apertura: la sanadora de las heridas y la que criticaba los excesos cometidos (Botton Beja, 2008, pp. 741-742).

Wang Xiaobo aborda una temática que, en teoría, ya había sido explotada a fondo por sus contemporáneos luego de la apertura de finales de la década de 1970. Por aquel entonces estaba en boga la llamada "literatura de cicatrices". En realidad, esa literatura ofrecía una perspectiva de la Revolución Cultural cargada de dramatismo y dirigía sus críticas exclusivamente a las desgracias provocadas por la Banda de los Cuatro. Esas obras, como afirma King (2013): “describen un mundo tomado por oportunistas mezquinos y rencorosos, que aterrorizan a gente mejor que ellos en nombre de la Dictadura del Proletariado" (p. 162). En otras palabras, ni se había llegado a criticar a otros líderes políticos, como el propio Mao Zedong, ni se había dado la adecuada atención a otros periodos funestos de la era maoísta, como las grandes campañas ideológicas de los años cincuenta o el Gran Salto Adelante (1958-1961). Por si fuera poco, eran obras que habían sido elaboradas en muchas ocasiones de forma burda y carentes de estilo. Antes de que el mundo literario -y la propia población china- hubiera tenido tiempo de reconciliarse como era debido con el pasado reciente y de dar una respuesta acorde con el horror y el disparate del maoísmo, ya habían surgido otras corrientes literarias durante la llamada "Fiebre Cultural" de los años ochenta.

El nombre de Wang Xiaobo comenzó a resonar en los círculos literarios a raíz del éxito alcanzado fuera de China continental, cuando le fueron otorgados los galardones de la crítica literaria taiwanesa, en 1991, por su novela 黄金时代 Huángjīn shídài [La edad de oro] (Wang, 1992) y, en 1995, por 未来世界 Weilái shijiè [El mundo futuro] (Wang, 1995). Wang Xiaobo adquirió además gran reputación debido a su producción ensayística. En 1996, un año antes de su muerte, se publicó una colección de ensayos titulada 思维的乐趣 Sìréi de lèqù [E] placer de pensar] (Wang, 1996b), y poco después de su falleci- 
miento, en 1997, se imprimió otra colección con el título de 沉默的大多数 Chénmò de dà duōshù [La mayoría silenciosa] (Wang, 1997b).

Conviene ahora poner de relieve varios aspectos o circunstancias que ayudaron a la popularización de la obra de Wang Xiaobo. Primeramente, fue el primer autor de China continental que ganó el premio literario otorgado por el diario taiwanés UDN por la mejor novela y el único que lo obtuvo en dos ocasiones; fue coautor - junto con su esposa, la socióloga Li Yinhe (n. 1952) - del primer estudio serio sobre la homosexualidad en China: 他们的世界 Tāmen de shijiè [Su mundo] (Li y Wang, 1992). Fue, asimismo, el primer guionista chino en obtener un galardón -junto con Yuan Zhang- al mejor guion en un festival internacional ${ }^{2}$ por la película 东宫西宫 Dōng gōng xī gōng [Palacio del Este, Palacio del Oeste] (Jung y Yuan, 1996; Berry, 2005).

En segundo término, Wang Xiaobo murió de forma repentina sin haber completado cinco años de dedicación exclusiva a la literatura. Había irrumpido en la escena de las letras chinas tras haberse forjado una reputación desde la periferia taiwanesa, sin contar siquiera con la mediación de los ć́rculos literarios del continente (Shernuk y Suher, 2017). Poco a poco, se había hecho con una base de lectores muy motivados. Su prematura y repentina muerte hizo que su obra se revalorase aún más y que se acrecentase su fama.

En tercer lugar, la mayor parte de la ficción de Wang Xiaobo tiene como marco los años de la Revolución Cultural, con menciones ocasionales al Gran Salto Adelante. Sin embargo, no deja que la Revolución Cultural se transforme en otro protagonista de sus relatos. Los temas que de verdad le preocupaban se resumen en la defensa de la libertad y de la razón, así como en los problemas a los que ha de hacer frente el individuo dentro de la sociedad. Para Wang Xiaobo, la Revolución Cultural

${ }^{2}$ Galardón otorgado en el XI Festival Internacional de Cine de Mar del Plata (Argentina) de 1996. 
es uno de los sucesos más negativos de la historia de China. Antes de considerar cualquier tema social o intelectual es indispensable comprender en profundidad tal Revolución; esa actitud es un requisito para mirar hacia el futuro (Wang, 1998).

Otro aspecto singular que ayuda a comprender la fama de Wang Xiaobo es su novedoso estilo de expresión. No cae en el dramatismo ni en el sentimentalismo exacerbado de sus coetáneos; mantiene más bien una postura distanciada ante el trauma y el horror. El conjunto de rasgos estilísticos que individualizan y concretan su originalidad, su estilo peculiar, su eficacia, en suma, se debe a particularidades de la vida del autor: poseía una gran cultura, fue educado en el seno de una familia de intelectuales y vivió y estudió en el extranjero, lo que permitió que pasara suficiente tiempo madurando lo que habría de ser su estilo y su visión del mundo literarios.

Su hermano mayor y biógrafo, Wang Xiaoping (n. 1949), asegura que el estilo de Mark Twain y Bernard Shaw influyó mucho en Wang Xiaobo (Wang, 2012), y de acuerdo con el propio Wang Xiaobo, lo mismo ocurrió con el clásico de George Orwell, que lo inspiró para escribir 白银时代 Báizín shídài [La edad de plata] (Wang, 1997a), novela en la que el futuro se presenta como una nueva proyección semidistorsionada del pasado. Para Wang Xiaobo no ha habido corte entre la era maoísta y la posterior, sino que todo ha seguido una progresión fluida, de un estado de estancamiento al siguiente, donde la presión ejercida por el sistema sobre sus personajes se vuelve tan sofocante que amenaza con deformar su subjetividad.

Wang Xiaobo es un acérrimo defensor del individualismo, así como un luchador contra las fuerzas alienantes de un poder establecido e incontrolable. Tanto Orwell como Kafka estuvieron en esa línea. El primero, en su novela 1984 (publicada en 1949), habla del triunfo del totalitarismo comunista en la vida de la gente en Inglaterra, vigilada por una "Policía del Pensamiento" y mediatizada por un Ministerio de la Verdad, que realiza una falsificación sistemática de la verdad mis- 
ma y que ha inventado un nuevo lenguaje hecho de neologismos y duplicidad, hasta el punto de que lo negro lo convierte en blanco. En medio de ese ambiente carcelario, sólo unos cuantos conservan un instinto de libertad. En otra de sus obras, Rebelión en la granja (1945), hizo una crítica alegórica y mordaz del totalitarismo, en especial el de corte comunista, con una buena carga de humor negro, además de potentes recursos estilísticos, como la alegoría y la personificación, algo de lo que hablo enseguida en relación con el ensayo "Un cerdo inconformista" de Wang Xiaobo. En cuanto a los paralelismos entre éste y Kafka, encontramos que los personajes creados por ambos se refugian en la introspección al verse inmersos en un mundo arduo y difícil de comprender, o ante una burocracia tiránica y agobiante. Esto hace imposible el desarrollo del individuo, como sucede en El castillo (1926), al que el agrimensor no puede acceder porque desconoce las reglas para enfrentar a un poder inescrutable y opresivo frente al cual el individuo se halla inerme.

Durante toda su infancia, Wang Xiaobo fue testigo de primera mano del acoso continuo a los intelectuales, ya que su propio padre y su círculo de amistades habían sido señalados durante las grandes campañas de movilización de las décadas de 1950 y 1960, si bien, en la adolescencia -y tras el inevitable adoctrinamiento-, se encontraba preparado y deseoso de ir a hacer la revolución con los demás "jóvenes instruidos". ${ }^{3}$

${ }^{3}$ La expresión “jóvenes instruidos” (知识青年 Zhishì qingnián, simplificado en 知 青 Zhiqing) era la manera de denominar a aquellos que, entre finales de los años sesenta y los setenta fueron enviados, bien por voluntad propia, bien siguiendo órdenes, a zonas rurales o fábricas para ser adscritos a equipos de trabajo. Durante la Revolución Cultural, alrededor de 17 millones de ellos fueron alejados así de los núcleos urbanos durante el llamado “Movimiento de subir a las montañas y bajar al campo" (上山下 乡运动 Shàngshān xià xiāng yùndòng). Entre sus filas se encontraba la mayoría de los guardias rojos, que habían llegado a causar grandes tumultos y destrozos en los núcleos urbanos. Mao, gracias a ellos, se deshizo con éxito de sus enemigos políticos; los jóvenes guardias habían cumplido su propósito y no era conveniente dejarlos campar a sus anchas con los demás adolescentes por las ciudades - los centros educativos habían sido clausurados y no había mucho que hacer-. Se les asignó, entonces, la 
Según afirma Wang Xiaoping (2012), la visión bucólica de la vida en la comuna que tenía Xiaobo se desvaneció poco después de haber llegado a Yunnan. Allí soportó largas jornadas de labor bajo el pesado clima subtropical con su brigada de trabajo, en un ambiente deshumanizado, sórdido y surrealista, donde las necesidades personales eran ignoradas y los individuos, alienados; donde el único sonido de fondo era una estridente cacofonía de alabanzas al comunismo. Sus experiencias en la provincia sureña de Yunnan, durante este periodo de la historia, lo llevaron a escribir, y posteriormente las retomó una y otra vez como componente básico para su obra; sin ir más lejos, su novela más célebre, 黄金时代 Huángjinn shídài [ $\mathrm{La}$ edad de oro], tiene como telón de fondo la Revolución Cultural.

Con una altura de $1.84 \mathrm{~m}$ y delicado de salud, Wang Xiaobo sufría constantes lumbalgias debido a las largas jornadas de labor en los campos de arroz. A esto se añadía la escasez de comida y las raras veces que veía un pedazo de carne. Según relata su hermano, Xiaobo y algunos compañeros comenzaron a robar pollos a los lugareños, pero fueron detenidos y llevados a la granja para ser sometidos a una sesión de humillación pública (Wang, 2012, p. 146). Peor aún era la sensación de aburrimiento y desolación que los invadía, y que los llevó a pensar que habían sido abandonados y olvidados (Wang, 1996b). Por la proximidad de la provincia de Yunnan a la frontera, algunos compañeros de Xiaobo optaron por fugarse a Birmania, bien fuera para dar asistencia al Partido Comunista birmano, bien fuera para vivir unos años de puro desenfreno, aunque aquello significara acortar su esperanza de vida. Wang Xiaobo se planteó esa opción, pero "tras ponderarla durante una noche y fumarse dos paquetes de tabaco, decidió no ir con ellos" (Wang, 2012, p. 148). Más adelante le llegarían noticias de sus compañeros fugados: la mayoría pereció en los bosques tropicales de Birmania.

misión de "aprender a hacer la revolución y desarrollar sus talentos al máximo" con los campesinos de clase pobre y media-baja. 
De vuelta a Beijing, Wang Xiaobo cultivó su estilo y produjo gran cantidad de escritos. Continuó formándose, primero en la Universidad de Renmin y más tarde en Estados Unidos, en la Universidad de Pittsburgh; con todo, sabedor de los problemas que le traería manifestar de manera pública sus ideas, permaneció en el anonimato durante años, mientras perfeccionaba sus obras.

A lo largo de la década de 1990, Wang Xiaobo pasó de ser un miembro de "la mayoría silenciosa" a un intelectual sin pelos en la lengua. En su carácter de escritor, Wang se propuso la misión de hacer frente al despotismo del Partido Comunista de China y de abogar por la libertad a fin de realizar los anhelos individuales en la sociedad china (Shernuk y Suher, 2017, p. 825).

\section{Sobre el ensayo "Un cerdo inconformista"}

Luego del esbozo de algunas circunstancias vivenciales de Wang Xiaobo, es preciso hablar del ensayo aquí traducido, cuyo asunto es el anhelo de libertad, la defensa de la individualidad $y$, en fin, la dificultad que supone actuar en contra de las imposiciones marcadas por el entorno. Este último punto adquiere capital importancia en una sociedad como la china, donde la mejor opción para el individuo es no destacar y en la que es muy difícil que se produzcan grandes cambios. Como tema secundario se encuentra la indolencia con la que la mayoría acepta que le sea impuesta una manera de vivir determinada, a lo que se alude con la vida de los "jóvenes instruidos" durante la Revolución Cultural, adoctrinados mediante eslóganes simplistas muy similares a los de aquellas atolondradas ovejas y gallinas que, en la Rebelión en la granja, lanzaban consignas a diestra y siniestra.

El ensayo "Un cerdo inconformista" es de suyo brillante y cargado de humor, quizá por eso sea uno de los más apreciados entre los lectores de Wang Xiaobo. En él se hace una analogía marcadamente orwelliana entre los animales de granja y la socie- 
dad planificada. En pocas palabras, los humanos son capaces de dirigir las vidas de otros humanos como si fueran animales, por ejemplo, los cerdos; de ahí que sea este animal el protagonista de esta historia: un "cerdo inconformista", que no se pliega a seguir las normas establecidas, aparece en el relato como un auténtico rebelde y un librepensador, dos características que adquieren más valor en una sociedad como la china actual, donde el individuo queda supeditado al grupo. Me ha parecido por demás curioso constatar cómo este mensaje ha calado con tanta fuerza entre los lectores, tanto en los blogs como en las discusiones en Internet, pues siempre hay alguien que desearía actuar como el cerdo inconformista. Por esta pieza, así como por otras muchas rubricadas por Wang Xiaobo, es posible concluir que su obra tuvo y tiene hoy considerable vigencia.

Conviene ahora analizar, siquiera de forma somera, esta pieza ensayística. Cuenta con cerca de 2000 caracteres y fue dada a la imprenta por primera vez el 15 de junio de 1996 en la revista 三联生活周刊 [Life Weekly] (Wang, 1996a). Tras la muerte de Wang Xiaobo fue publicada de nuevo en 2005, en el recopilatorio de ensayos titulado Siwei de lequ, 思维的乐趣 [El placer de pensar]. A todas luces, es uno de los escritos más famosos de este autor.

"Un cerdo inconformista" es un ensayo expositivo-descriptivo con una estructura estándar y una argumentación interna de naturaleza inductiva. El autor comienza dando cuenta de un estado de cosas, luego presenta una serie de hechos y fenómenos para rematar el escrito con una simple observación a modo de conclusión, todo englobado en la anécdota que se narra. Las ideas generales del tema han de ser inferidas por el lector. La actitud frente a la realidad es, en su mayor parte, externa y descriptiva. La postura del autor es lógica y realista, si bien no estrictamente objetiva, ya que contiene algún juicio de valor, así como comentarios en clave de humor negro e ironía crítica: "Los líderes [...] lo tildaron de elemento maligno [...] y quisieron aplicarle métodos de la dictadura del proletariado". Las 
descripciones se producen, sobre todo, desde el punto de vista de la primera persona en forma de narrador testigo, aunque hay también un uso muy pronunciado de la tercera persona. En una ocasión se define el grado de cercanía con el lector por medio de un giro a segunda persona: "si ibas a intentarlo, aunque escondieras el cuchillo [...] se te quedaba mirando". Su disposición en la transmisión es básicamente realista. Destaca la profusión de sustantivos, en especial los concretos, lo que hace que el discurso contenga un alto grado de realismo y fluidez. Los verbos son muy abundantes, por lo común en modo indicativo, cuyo efecto es que la argumentación se instale en la realidad. El registro es coloquial, a fin de enfatizar la acción y aportar dinamismo a la descripción de los hechos. Es muy evidente la ausencia de adjetivos, hay poco más de una treintena en todo el texto, la mayoría nada rebuscados; con ello, el autor ni embellece ni valora, sino que se centra en la acción. En el plano sintáctico predominan las oraciones complejas. Llama la atención, en efecto, el gran número de oraciones coordinadas, sobre todo las adversativas, las explicativas, las consecutivas y las yuxtapuestas, lo que resulta en una lectura ágil del texto. Asimismo, destacan las condicionales, debido a la naturaleza inductiva de la argumentación, oraciones muy características en la ensayística de Wang Xiaobo.

En la traducción me he atenido, en esencia, a la literalidad del original en lugar de optar por un resultado más literario o artístico, pues de haber elegido esta segunda opción, se habrían perdido no pocos matices de la intención comunicativa de Wang Xiaobo y se vería reducido el impacto pretendido. Creo haber realizado una versión fiel y ajustada al texto, puesto que, si ciertamente era deseable hacer una traducción legible e idiomática, era necesario ante todo efectuar un traslado lo más ceñido posible al texto original y evitar traducir aquellas voces o expresiones que tienen un sentido específico en la sociedad que las emplea, mediante términos no del todo equivalentes o inadecuados.

Luego del traslado inicial de la pieza, hice cierto número de revisiones, con especial énfasis en las estructuras gramaticales 
del texto en chino, a fin de que en la versión en español no se hubiera desvirtuado el tiempo o el aspecto originales. Completadas las dos primeras fases, procedí a ajustar la traducción a una versión más inteligible, buscando siempre el equilibrio para no perder la frescura del peculiar estilo del autor. Fue necesario resolver en cada párrafo problemas prácticos relacionados con las formidables diferencias entre la lengua de origen y la de destino. En especial, destaca la alta frecuencia de adverbios como 都, 就, 还 y 当然, de los demostrativos 这 y 那, y de conectores discursivos como 但 o 却. Todos son comunes en el registro coloquial en chino y se emplean, por lo general, para dar dinamismo al discurso; no obstante, hubiera sido muy pesado mantener el mismo número en la traducción al español. En la medida de lo posible, mantuve estas palabras y locuciones, si bien las distribuí en el texto y utilicé sinónimos; por ejemplo, traduje 但 a veces como "pero", "sin embargo", "no obstante", "con todo", etcétera.

Respeté, siempre que fue aceptable, el orden de los sintagmas dentro de las oraciones en chino, por ello recurrí al hipérbaton en mi versión española, aprovechando la alta movilidad que tienen en nuestra lengua el adverbio, el adjetivo e incluso el verbo. En cuanto a las frases hechas y los refranes, tuve que juzgar en mis revisiones si correspondía traducirlos de manera literal o con un equivalente en nuestra lengua, o si era necesaria una nota al pie.

Parece pertinente, llegados aquí, ofrecer traducido al español el ensayo en cuestión.

\section{Referencias}

Berry, M. (2005). Wang Xiaobo. En E. L. Davis (Ed.), Encyclopedia of Contemporary Chinese Culture (p. 889). Nueva York, NY: Routledge.

Botton Beja, F. (2008). Tendencias de la literatura china moderna. En R. Cornejo (Ed.), China: Radiografía de una potencia en ascenso (pp. 737-750). México: El Colegio de México. 
Jung C. (Prod.) y Yuan Z. (Dir.) (1996). 东宫西宫 Dong gong xi gong [Palacio del Este, Palacio del Oeste] [cinta cinematográfica]. China: Amazon Entertainment Ltd.

KAFKA, F. (2003). El castillo. Madrid: Cátedra.

KING, R. (2013). Milestones on a golden road: Writing for Chinese socialism 1945-80. Vancouver: UBC Press.

Li Y. y Wang X. 李银河 王曉波 (1992). Tamen de Shijie - Zhongguo nan tongxinlian qunluo toushi 他们的世界——中国男同性恋群 落透视 [Su mundo: perspectivas sobre la comunidad homosexual en China]. Taiyuan: Shanxi Renmin Chubanshe.

Orwell, G. (2010). 1984. Barcelona: Destino.

ORWeLl, G. (2010). Rebelión en la granja. Barcelona: Austral.

SHERNUK, K. y Suher, D. (2017). From the margins to the mainstream: A tale of two Wangs. En D. Der-wei Wang (Ed.), A Nere literary history of modern China (pp. 821-826). Cambridge, MA: The Belknap Press of Harvard University Press. https://doi. org/10.4159/9780674978898-141

WANG X. 王小波 (1992). Huángiinn shídài 黄金时代 [La edad de oro].

Taipéi: Taiwan Lian jing Chubanshe.

WANG X. 王小波 (1995). Weilai Shijie 未来世界 [El mundo futuro].

Taipéi: Taiwan Lian jing Chubanshe.

WANG X. 王小波 (1996a). Yi zhi duli duxing de zhu 只特立独行的

猪 [Un cerdo inconformista]. Life Weekly (11).

WANG X. 王小波 (1996b). Siwei de lequ 思维的乐趣 [El placer de pensar]. Taiyuan: Beiyue Wenyi Chubanshe.

WANG X. 王小波 (1997a). Báiyín shídài (白银时代) [La edad de plata]. Guangzhou: Huacheng Chubanshe.

WANG X. 王小波 (1997b). Chenmo de daduoshu 沉默的大多数 [La mayoría silenciosa]. Beijing: Zhongguo Qingnian Chubanshe.

WANG X. 王小波 (1998). Heitie shidài 黑铁时代 [La edad de hierro]. Changchún: Shidai Wenyi Chubanshe.

WANG X. 王小平 (2012). Wo de xiongdi Wang Xiaobo 我的兄弟 王小波 [Mi hermano Wang Xiaobo]. Nankín: Jiangsu Wenyi Chubanshe. 
Ismael A. Maíllo es licenciado en estudios chinos por la Universidad de Durham y doctor en lenguas modernas (chino) por la Universidad de Salamanca. Tras haber vivido en Beijing durante diez años para ampliar sus estudios, ejerció como profesor de chino mandarín en la Escuela Oficial de Idiomas de Valladolid. En la actualidad es profesor en la Universidad de Salamanca, en la que imparte lengua, historia y literatura chinas; asimismo, es coordinador de lengua china en el grado en estudios de Asia Oriental de la misma universidad.

http://orcid.org/0000-0003-2578-6495 imaillo@usal.es 


\section{Un cerdo inconformista}

Durante mis tiempos en la comuna, he echado de comer a los cerdos y también he sacado las vacas a pastar. Si no hay nadie que los cuide, estas dos especies de animales también saben de sobra cómo arreglárselas. Pueden pasearse a su aire; si hay hambre comen pues, y si hay sed, entonces beben; al llegar la primavera incluso se dan al cortejo: ${ }^{4}$ de esta manera, su nivel de vida es muy primario, no hay en absoluto nada que destacar. Al llegar el hombre, sus vidas fueron reorganizadas: la vida de cada vaca y cada cerdo tenía ahora objeto. Y es que, hablando de la mayoría de ellos, el objeto de sus vidas es muy trágico: el objeto de los primeros es trabajar, el de los segundos es engordar. No creo que tuvieran nada de qué quejarse, porque mi vida en aquel momento tampoco es que fuera mucho más variada, aparte de las "ocho óperas modelo", ${ }^{5}$ no había ningún otro pasatiempo. Había un número escaso en extremo de cerdos y vacas; sus vidas tenían otro arreglo.

Tomando los cerdos como ejemplo, los verracos y las cerdas nodrizas, aparte de comer, tenían otras cosas que hacer. Por lo que yo he visto, a éstos tampoco es que les gustaran mucho tales arreglos. La misión de los verracos era copular; en otras palabras, nuestras políticas los autorizaban a ser unos picaflores. Sin embargo, los verracos agotados adoptaban a menudo un aire de caballerosidad que sólo los cebones tenían (los cebones son los que están capados), y ni muertos querían montar a las

${ }^{4}$ Lit. "hablar de amor".

${ }^{5}$ Durante la Revolución Cultural (1966-1976), la ópera china tradicional fue proscrita y apartada. Sólo fueron autorizadas las “ocho obras modelo” (八个样板 戏 bā ge yàngbăn xì), compuestas por cinco óperas, dos ballets y una sinfonía. Estas piezas, promovidas por la mujer de Mao, Jiang Qing (1914-1991), se centraban en los trabajadores y los campesinos y se representaban en el estilo tradicional operístico, aunque sin el vestuario elegante de épocas anteriores. Durante aquellos años fueron las únicas obras representadas en todo el país. La expresión popular "ocho obras modelo para ochocientos millones de chinos” describe aquella falta de variedad. 
marranas. La misión de las cerdas nodrizas era engendrar crías, pero había algunas marranas que se comían a las crías. Resumiendo, la organización de los seres humanos provocaba en los cerdos un sufrimiento insoportable. Pero ellos lo aguantaban de todos modos: los cerdos siguen siendo cerdos.

Hacerle a la vida toda clase de ajustes es una característica propia del hombre. No sólo se la regula a los animales, también a sí mismo. Sabemos que en la antigua Grecia existía Esparta, y la vida allí fue regulada hasta el punto de que se había perdido la alegría de vivir; la meta era precisamente hacer que los hombres se convirtieran en guerreros dispuestos a morir, y hacer que las mujeres se volvieran máquinas de procrear. Los primeros parecían gallos de pelea; las segundas parecían cerdas nodrizas. Estas dos categorías de animales eran muy especiales, pero creo que a ellos seguro que no les gustaba su propia vida. Pero, aunque no guste, ¿qué se le va a hacer? Sean personas o animales da igual, es muy difícil cambiar su propio destino.

A continuación, se va a hablar de un cerdo que era un tanto diferente a los demás. Cuando yo le echaba de comer a los cerdos, él ya tendría cuatro o cinco años. Tomando en cuenta su estatus, era cerdo para carne; no obstante, era a la vez negro y enjuto y sus ojos centelleaban. Este colega era ágil como una cabra: las vallas de un metro de alto de las pocilgas las pasaba de un salto, e incluso podía saltar al tejado del chozo de los cerdos; en ese punto parecía ya un gato, por eso siempre se iba a hacer turismo por todas partes, de ninguna manera se quedaba en la pocilga. Todos los “jóvenes instruidos” que habían tenido que alimentar a los cerdos lo trataban como si fuera una mascota; también era mi mascota, porque él sólo era bueno con los "jóvenes instruidos", y permitía que se le acercaran a menos de tres metros; de ser otra gente, él habría salido corriendo mucho antes.

Era macho y, en principio, debería haber sido capado. Pero si ibas a intentarlo, aunque escondieras el cuchillo de capar a tu espalda, él podía olerlo; se te quedaba mirando con los ojos muy 
abiertos y empezaba a gruñir: joinc, oinc! Yo siempre le echaba de comer unas gachas de salvado de trigo fino, y me esperaba a que se hubiera saciado para mezclar el salvado con hierbajos y alimentar a los demás cerdos. Los otros cerdos, al verlo, se ponían celosos y comenzaban todos juntos a gruñir. En esos momentos toda la pocilga era un griterío de mil demonios, ${ }^{6}$ pero yo y él, ni caso. Después de hartarse a comer, saltaba al tejado a tomar el sol o imitaba toda clase de sonidos. Él sabía imitar el ruido de los coches, de los tractores, y lo hacía tal cual. A veces no había ni rastro de él en todo el día. Yo me figuraba que había ido a los pueblos cercanos a buscar hembras. Aquí, donde nosotros, también teníamos marranas, todas encerradas en las pocilgas, pero al haber sido usadas demasiado para procrear, habían perdido sus hechuras, estaban sucias y apestosas, a él no le interesaban; las cerdas de los pueblos eran más bonitas. Él contaba con muchas proezas extraordinarias, pero el periodo en que alimenté a los cerdos fue corto, y lo que llegué a saber es limitado, así que por eso no escribo más.

En resumen, a todos los “jóvenes instruidos” que habíamos echado de comer a los cerdos nos caía bien, nos gustaba su estilo inconformista y hasta decíamos que vivía desinhibido. Los campesinos no eran tan románticos; decían que este cerdo era un indecente. Los líderes lo detestaban; sobre este punto ya hablaremos más adelante. Sin embargo, a mí no sólo es que me gustara, lo veneraba; a menudo, sin importarme el hecho de que yo fuera más de diez años mayor que él, lo llamaba "hermano mayor cerdo". Como dije, este hermano mayor cerdo podía imitar toda clase de sonidos. Yo creo que él también estudió el lenguaje humano, pero no consiguió aprenderlo; de haberlo aprendido, podríamos haber mantenido conversaciones con el corazón en la mano. Pero esto no se le podía reprochar, el timbre de los cerdos y el de los hombres difiere demasiado.

${ }^{6}$ Lit. “un griterío de espíritus y aullido de lobos” (鬼哭狼嚎 gǔ̌ku lángháo). 
Después, el hermano cerdo aprendió a imitar el sonido de las sirenas; esta habilidad le acarreó problemas. Allí donde estábamos había una fábrica azucarera en la que, a mediodía, sonaba una vez la sirena para que los obreros cambiaran de turno. Nuestro equipo, cuando iba a trabajar al campo, al escuchar el sonido de esta sirena paraba de trabajar y marchaba de vuelta. Mi hermano cerdo cada mañana, a las diez en punto, saltaba al tejado a imitar la sirena. La gente que estaba en el campo, al oírlo se volvía - esto era una hora y media más temprano que la sirena de la azucarera-. A decir verdad, no se podía culpar de todo esto al hermano cerdo, al fin y al cabo, él no era una caldera, y entre sus chillidos y el sonido de la sirena sí que había algo de diferencia, pero los pueblerinos se obstinaban en decir que no los diferenciaban.

Los líderes, por su lado, se reunieron debido a esto y lo tildaron de elemento maligno y dañino para la labranza de primavera, y quisieron aplicarle métodos de la dictadura del proletariado - yo ya conocía lo esencial de la reunión, pero no me preocupé por él-, porque si la dictadura del proletariado señalaba la soga y el cuchillo de matar cerdos, entonces no tendría ninguna posibilidad. Los líderes anteriores tampoco es que no lo hubieran intentado; ni cien personas consiguieron atraparlo. Los perros tampoco servían: el hermano cerdo, al correr, parecía un torpedo, podía embestir a los perros y mandarlos volando a tres metros.

Quién iba a saber que esta vez sí que actuarían en serio. $\mathrm{El}$ instructor político trajo veintitantas personas armadas con pistolas modelo 54; el viceinstructor político trajo más de diez, provistas de fusiles con los que vigilaban los sembrados. Se dividieron en dos grupos y lo acorralaron en el terreno vacío fuera de la pocilga. Esto hizo que me abismase en un dilema en mi fuero interno: teniendo en cuenta mi amistad con él, debería haberme lanzado a toda prisa blandiendo dos cuchillos de matar cerdos y luchar codo a codo con él, pero al mismo tiempo me parecía que actuar de esta manera habría escandalizado demasiado a todo el mundo; al fin y al cabo, no 
era más que un cerdo. Había otra razón, además: no me atrevía a oponerme a los líderes; me temo que éste era el quid de la cuestión. Total que me quedé a un lado mirando.

La compostura del hermano cerdo me hacía admirarlo muchísimo: muy fríamente se refugió entre las líneas de fuego de las pistolas y los fusiles y dejó que la gente gritara y los perros lo mordieran sin abandonar esa posición. De esta manera, si los que empuñaban pistolas disparaban, podían matar a los que llevaban fusiles y viceversa; de abrir fuego al mismo tiempo, ambos bandos se habrían matado. En cuanto a él, al ser un blanco pequeño, probablemente no le pasaría nada. Y, de esta forma, dando varias vueltas seguidas, encontró un hueco y salió de una embestida corriendo con extrema soltura.

Más adelante lo volví a ver una vez más en los campos de caña de azúcar; le habían crecido los colmillos y todavía me conocía, pero ya no me dejaba acercármele. Esta frialdad me dolía, pero yo también aprobaba que guardara las distancias con gente malintencionada.

Yo ya tengo cuarenta años y, aparte de este cerdo, todavía no he visto a nadie que se atreviera a ignorar así las imposiciones de la vida. Al contrario, he visto a mucha gente que quería estructurar la existencia de otros; también hay gente que sobrelleva con indolencia que se les haya planificado la existencia. Por esta razón, siempre recuerdo con nostalgia a ese cerdo inconformista. 